\title{
Treatment Options in the Management of Thrombocytopenia in Patients Infected with HCV
}

\author{
André Cosme de Oliveira \\ Liver Transplant Section of the Clinical Hospital of University of São Paulo School of Medicine (HC-FMUSP); São Paulo, SP, Brazil
}

Thrombocytopenia (platelet count $<80,000 / \mathrm{mm}^{3}$ ) in patients with chronic hepatitis has been associated with two factors: the first is hypersplenism resulting from splenomegaly in portal hypertension [1]. The spleen continuously sequesters one-third of circulating platelets, so that splenomegaly increases the fraction of platelets trapped in the splenic sinusoids, especially when resulting from passive congestion or an increase in venous portal pressure [1]. Hypersplenism seems to be the most common cause of thrombocytopenia associated with liver cirrhosis and portal hypertension.

The second mechanism is related to the decreased production of thrombopoietin, a hormone produced by hepatocytes, which regulates the development of the megakaryocyte. In cirrhosis, due to the reduction in the mass of functioning hepatocytes, there can be a reduction of thrombopoiesis in the bone marrow, leading to thrombocytopenia in the peripheral blood [1].

In some situations, patients who are otherwise eligible for $\mathrm{HCV}$ treatment with interferon and ribavirin cannot be so treated because their platelets counts are low, which jeopardizes the treatment $[2,3]$.

Nevertheless, hepatitis $C$ patients treated with interferon and ribavirin also present a drop in the platelet count as a side effect $[2,3]$.

There is as yet no treatment consensus in the literature for the management of these patients. We therefore present some related studies that address the management of these patients, in the pre-treatment and intra-treatment phases.

Patients receiving interferon alpha or peginterferon alpha can present a 30-50\% reduction in the baseline platelet count, and a dose reduction is necessary in approximately $4 \%$ of the patients $[2,3]$. We should consider possible reduction of the dose when platelet counts drop to $<50,000 / \mathrm{mm}^{3}$, and discontinuation of treatment when $<25,000 / \mathrm{mm}^{3}$.

When using peginterferon alpha-2, the weekly dose can be reduced from $180 \mu \mathrm{g}$ to $135 \mu \mathrm{g}$, or even to $90 \mu \mathrm{g}$. While using peginterferon alpha- $2 \mathrm{~b}$, the weekly dose can be reduced from $1.5 \mu \mathrm{g} / \mathrm{kg}$ to $1.0 \mu \mathrm{g} / \mathrm{kg}$, or even to $0.5 \mu \mathrm{g} / \mathrm{kg}[2,3]$.

Human recombinant interleukin (IL)-11 (oprelvekin, Neumega ${ }^{\circledR}$; Wyeth Laboratories, Collegeville, PA, USA) can be used as a way to stimulate the increase in the number of platelets at a dose of $5 \mu \mathrm{g} / \mathrm{kg} /$ day, subcutaneously, initially for 7 days. If necessary, maintenance can consist of 1 to 3 doses per week during HCV drug therapy, similarly to what is recommended for filgrastim (granulocyte colony-stimulating factor) [4].

The Brazilian Journal of Infectious Diseases $\quad$ 2007;11 (5) Suppl. 1:71-72. (C) 2007 by The Brazilian Journal of Infectious Diseases and Contexto Publishing. All rights reserved.
Administration of IL-11 has multiple effects on the hematopoietic system, specifically promoting the proliferation and maturation of megakaryocytes and inducement of neutrophilia and thrombocytosis [5].

In patients with cirrhosis, its use should be restricted to those with Child-Pugh class A or B cirrhosis [5].

In a study conducted by Ghalib et al. [6], none of the patients presented increased platelet counts until day 4 of daily use of the medication, and the maximal therapeutic response was observed near day 13 (Figure 1). At 6 to 8 days after the initiation of treatment, $89 \%$ of the patients presented duplication of the initial number of platelets, and $78 \%$ of the patients presented platelet counts $>80,000 / \mathrm{mm}^{3}$.

Another treatment option for thrombocytopenia in these patients is splenic embolization.

Figure 1. Ratio between platelet counts and duration of treatment with interleukin-11.

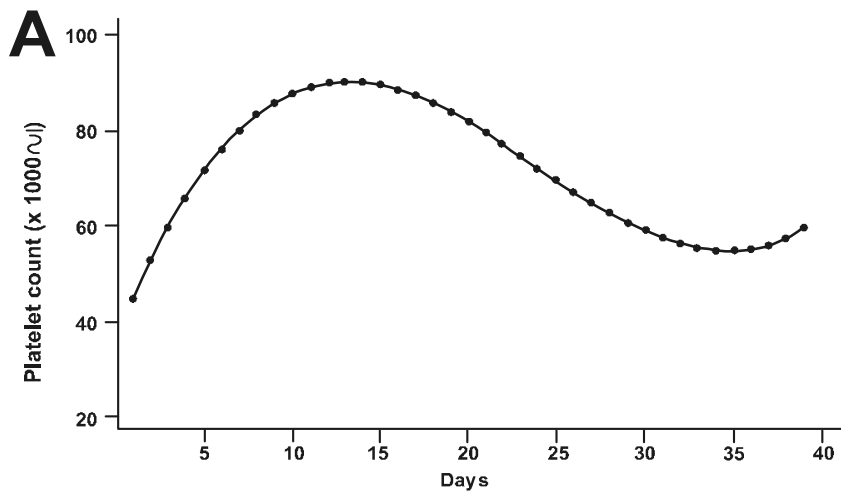

Splenic embolization should preferably be performed with injection of polyvinyl alcohol particles between 355 and 500 $\mu \mathrm{m}$ (Trufill PVA Embolization Particles; Cordis Corp., Johnson \& Johnson Co., Miami Lakes, FL, USA) into the splenic artery and should not exceed an embolization volume of $30 \%$ of the initial spleen volume [7].

The most severe complications after embolization can be ascites, thrombosis of the splenic vein or portal vein, spontaneous bacterial peritonitis, and splenic abscess. Abdominal pain and fever are typically present postembolization [8].

Splenic embolization presented $50 \%$ of complication in embolizations $<30 \%$ of splenic volume, whereas, in embolizations $>30 \%, 100 \%$ of the patients presented complications [9].

Abdominal computed tomography scans can be performed 2 to 3 weeks after embolization for assessment of effectiveness of the procedure and to identify complications [10]. 
All patients should be previously vaccinated against pneumococci before embolization. In addition, antibiotic therapy should be initiated and maintained during hospitalization of the patient. We suggest the use of cefotaxime and ampicillin in these cases [11]. The combined treatment with interferon and ribavirin should not be initiated or reinitiated until 8 weeks after splenic embolization [12].

The use of human recombinant interleukin-11 (IL-11) (oprelvekin) and splenic embolization are both feasible treatments for these patients, taking into account the characteristics of the individual health care facilities at which the follow-up treatment is administered.

\section{References}

1. Moreno A., Bárcena R., Blázquez J., et al. Thrombocytopenia can be found in patients with chronic hepatitis related to hepatitis $\mathrm{C}$ virus (HCV). Both hypersplenism and decreased liver production of thrombopoietin (TPO) have been hypothesized as mechanisms responsible for thrombocytopenia. Hospital Ramón y Cajal, Madrid, Spain.

2. Manns M.P., et al. Peginterferon alfa-2b plus ribavirin compared with interferon alfa-2b plus ribavirin for initial treatment of chronic hepatitis C: a randomised trial. Lancet 2001;358:958-65.

3. Fried M.W. Peginterferon alfa-2a plus ribavirin for chronic hepatitis C virus infection. N Engl J Med 2002;347:975-82.

4. Hennepin County Medical Center. Management of HCV and treatment side effects. April 10, 2005.
5. Lawitz E., Hepburn M., Casey T. A Pilot Study of Interleukin11 in Subjects with Chronic Hepatitis C and Advanced Liver Disease Nonresponsive to Antiviral Therapy. Gastroenterology Service; Infectious Diseases Service; Pathology Department; Brooke Army Medical Center, San Antonio, Texas.

6. Ghalib R., Levine C., Hassan M., et al. Recombinant Human Interleukin-11 Improves Thrombocytopenia in Patients With Cirrhosis. Hepatology 2003:1165-71.

7. Zhu K., Meng X., Li Z., et al. Partial splenic embolization using polyvinyl alcohol particles for hypersplenism in cirrhosis: A prospective randomized study. Eur J Radiol 2007;24.

8. Lee C.M., Leung T.K., Wang H.J., et al. Evaluation of the effect of partial splenic embolization on platelet values for liver cirrhosis patients with thrombocytopenia. World J Gastroenterol 2007;13(4):619-22.

9. Hayashi H., Beppu T., Masuda T., et al. Predictive factors for platelet increase after partial splenic embolization in liver cirrhosis patients. J Gastroenterol Hepatol 2007;7.

10. Lee C.M., Leung T.K., Wang H.J., et al. Evaluation of the effect of partial splenic embolization on platelet values for liver cirrhosis patients with thrombocytopenia. World J Gastroenterol 2007;13(4):619-22.

11. Foruny J.R., Blázquez J., Moreno A., et al. Safe use of pegylated interferon/ribavirin in hepatitis $\mathrm{C}$ virus cirrhotic patients with hypersplenism after partial splenic embolization. Eur J Gastroenterol Hepatol 2005;17(11):1157-64.

12. Pålsson B., Verbaan H. Partial splenic embolization as pretreatment for antiviral therapy in hepatitis C virus infection. Eur J Gastroenterol Hepatol 2005;17(11):1153-5. 